DOI: http://dx.doi.org/10.12957/demetra.2015.12981

\title{
Tamanho da porção e gordura trans: os rótulos de alimentos industrializados brasileiros estão adequados?
}

\author{
Serving size and trans-fat: are the brazilian nutrition labels adequate?
}

\author{
Nathalie Kliemann' \\ Mariana Vieira dos Santos Kraemer' \\ Bruna Maria Silveira' \\ David Alejandro González-Chica² \\ Rossana Pacheco da Costa Proença ${ }^{1}$ \\ ' Universidade Federal de Santa Catarina, \\ Núcleo de Pesquisa de Nutrição em Produção \\ de Refeições, Programa de Pós-Graduação \\ em Nutrição, Departamento de Nutrição. \\ Florianópolis-SC, Brasil. \\ 2 Universidade Federal de Santa Catarina, \\ Programa de Pós-Graduação em Nutrição, \\ Departamento de Nutrição, Florianópolis-SC, \\ Brasil. \\ Financiamento: Coordenação de Aperfeiçoamento \\ de Pessoal de Nível Superior (CAPES) - \\ concessão de Bolsas de mestrado, TDI 3 e PIBIC \\ Correspondência / Corrrespondence \\ Rossana Pacheco da Costa Proença \\ Departamento de Nutrição, Programa de Pós- \\ Graduação em Nutrição, Universidade Federal de \\ Santa Catarina, Campus Universitário, Trindade, \\ 88040-900 Florianópolis-SC, Brasil. \\ E-mail: rossana.costa@ufsc.br
}

\section{Resumo}

Objetivo: Associar o tamanho da porção com a presença de gordura trans em rótulos de alimentos industrializados comercializados em supermercado brasileiro. Metodologia: A porção foi categorizada de acordo com a legislação e a presença de gordura trans foi estimada pela notificação de componentes com gordura trans na lista de ingredientes. Estimou-se a prevalência de falsos negativos, considerando os produtos que na informação nutricional reportaram não ter gordura trans, mas que apresentavam componentes com gordura trans na lista de ingredientes. Foram utilizados os testes Qui-quadrado e Anova, sendo o valor-p $<0,05$ significativo. Resultados: Metade dos alimentos tinha gordura trans na lista de ingredientes e, em comparação com a informação nutricional, cerca de $40 \%$ eram falsos negativos. Os percentuais de gordura trans e de falsos negativos se incrementaram até o tamanho máximo permitido para porção, diminuindo entre aqueles com porção acima do permitido. Nos produtos prontos para o consumo, observou-se padrão similar entre a densidade energética e a presença de gordura trans na lista de ingredientes; no entanto, para o percentual de falsos negativos, esta relação foi inversa. Conclusões: A porção declarada nos rótulos pode estar associada à notificação de presença de gordura trans na informação nutricional.

Palavras-chave: Ácidos Graxos Trans. Rotulagem de Alimentos. Porção de Alimento. Informação Nutricional. 


\section{Abstract}

Objective: To associate serving size and trans fat on nutrition facts of packaged foods commercialized in a Brazilian supermarket. Methods: The serving size was categorized according the Brazilian law and the trans fat occurrence was determined by the ingredient list notification of hydrogenated fats designations. The false negative prevalence was estimated considering the products that reported the nonoccurrence of hydrogenated fat on nutrition facts, but contained this fat in the ingredient list. Chi-square and ANOVA were tested, considering as a statistically significant value $\mathrm{p}<0.05$. Results: Half of the evaluated products presented trans fat on the ingredient list and, comparing to the nutrition facts, almost $40 \%$ were false negative. The trans fat and false negative percentage increase with the serving size until the highest bounds of the variability permitted and, decrease between those products with serving size above the limit permitted. For products classified as ready for consumption, a similar pattern of energy density and the occurrence of trans fat on the ingredient list was observed; however, for the percentage of false negatives, this association was reversed. Conclusions: The information of serving size can be related to the occurrence of trans fat on nutrition facts of packaged foods.

Key words: Trans Fatty Acids. Food Labeling. Food Serving Size. Nutritional Facts.

\section{Introdução}

Nas últimas décadas, estudos vêm demonstrando mudanças no padrão alimentar no mundo e no Brasil, ${ }^{2}$ com substituição de grãos e cereais por alimentos de origem animal, açúcares, gorduras, e sobretudo alimentos industrializados. Pesquisadores propuseram, inclusive, a definição de alimentos ultraprocessados para aqueles modificados de forma a adicionar ou introduzir substâncias que alterem consistentemente sua natureza, relacionando o consumo desses alimentos principalmente com a atual epidemia de obesidade. ${ }^{3}$

Nesse contexto, muitos dos alimentos industrializados em geral apresentam elevadas quantidades de gordura trans. ${ }^{3,4} \mathrm{~A}$ indústria utiliza esse tipo de gordura, mediante processo de hidrogenação, para atribuir melhoria nas características físicas, sensoriais e aumentar a conservação dos alimentos industrializados. ${ }^{5}$ Contudo, o consumo de gordura trans representa um fator de risco para o desenvolvimento de diversas doenças crônicas como, por exemplo, doenças cardiovasculares e obesidade. ${ }^{6-8}$ 
Assim, considerando que o uso da gordura trans de origem industrial apresenta potenciais efeitos deletérios para a saúde, a Organização Mundial da Saúde (OMS) lançou, em 2004, a Estratégia Global para Promoção da Alimentação Saudável, Atividade Física e Saúde, estabelecendo como meta a eliminação do consumo de gordura trans industrial. ${ }^{9}$ Essa recomendação foi reforçada pela OMS em 2013, quando a relacionou ao combate às doenças crônicas não transmissíveis. ${ }^{10}$ A partir do início desse movimento, em 2008 a Organização Pan-Americana da Saúde (PAHO/ WHO), juntamente com órgãos de representação da indústria de alimentos, acordaram reduzir as quantidades de gordura trans dos produtos industrializados na América Latina e Caribe. ${ }^{11}$ Recentemente, a gordura trans foi citada como um dos pontos importantes abordados em artigos sobre os desafios para enfrentar a obesidade..$^{12,13}$

Com o intuito de informar o consumidor sobre a presença de gordura trans nos alimentos industrializados, a legislação brasileira, harmonizada para os países do Mercosul, inclui desde 2003 esta notificação como item obrigatório na rotulagem nutricional. ${ }^{14}$ A RDC nº 360/2003 tornou obrigatória a declaração do conteúdo de gorduras trans em relação à porção nos rótulos dos alimentos industrializados. Contudo, a resolução ${ }^{14}$ estabelece que possam ser considerados e divulgados como "zero trans" os alimentos que apresentarem teor de gorduras trans menor ou igual a 0,2 gramas na porção, valor descrito como não significativo nesta resolução.

Proença \& Silveira ${ }^{15}$ relatam que não está clara a fundamentação científica para o estabelecimento desses limites legais para notificação de gordura trans nos rótulos, bem como não estão de acordo com a recomendação da $\mathrm{OMS}^{9}$ de eliminação da gordura trans dos alimentos industrializados. Além disso, as autoras destacam que não há valores de recomendação de consumo para gordura trans, pois esse tipo de gordura não é um nutriente e pode trazer malefícios para a saúde. ${ }^{6,8}$ Refletem ainda que a determinação de um limite para a declaração de gordura trans nos rótulos fragiliza a legislação, pois a indicação de ausência de gordura trans na informação nutricional não é segura e exige que o consumidor leia a lista de ingredientes para ter maior possibilidade de identificar a presença de gordura trans no alimento. ${ }^{15}$

Hissanaga et al. ${ }^{16,17}$ analisaram os rótulos de alimentos industrializados brasileiros destacados como isentos de gordura trans e encontram na maioria deles a declaração de componentes fontes de gordura trans na lista de ingredientes. Gagliardi et al. ${ }^{18}$ analisaram laboratorialmente a presença de gordura trans em alimentos industrializados do tipo fast food brasileiros com alegação de $0 \%$ deste ingrediente e detectaram presença de gordura trans em todos os alimentos avaliados, com valores que oscilaram de $0,01 \mathrm{~g}$ a 1,97g de gordura trans por porção.

O tamanho da porção de referência para rotulagem em gramas (g) ou mililitros (ml) é regulamentado pela RDC n⿳ 359/2003, ${ }^{19}$ que permite que sejam declaradas porções até $30 \%$ maiores ou menores do que o valor recomendado. Entretanto, alguns alimentos industrializados não apresentam uma porção de referência nesta legislação, como é o caso dos pratos prontos para 
o consumo. Para estes produtos, a legislação determina que a porção seja a quantidade em gramas correspondente a até 500 kilocalorias (kcal) do produto. ${ }^{19}$ Sendo assim, a indústria de alimentos tem a possibilidade de escolher diferentes valores de porção para rotular alimentos industrializados, o que pode influenciar a informação disponibilizada sobre a quantidade de nutrientes presentes no produto. De acordo com Proença \& Silveira, ${ }^{15}$ quando a quantidade de gordura trans não alcança o limite de 0,2 gramas por porção preconizada pela legislação, a empresa fica desobrigada de disponibilizar a quantidade dessa gordura no rótulo dos alimentos.

Dessa forma, considerando a hipótese de que o tamanho da porção pode ser determinante na notificação de gordura trans no rótulo, esta pesquisa objetivou analisar a associação entre o tamanho da porção e a presença de gordura trans notificada em alimentos industrializados brasileiros, considerando as informações do rótulo disponíveis aos consumidores.

\section{Metodologia}

\section{Coleta de dados}

Foi realizado estudo transversal no qual foram analisados todos os alimentos industrializados disponíveis à venda em um grande supermercado de Florianópolis-SC, capital de estado no sul do Brasil. Foi escolhido aleatoriamente um dos supermercados pertencente a uma rede com 21 lojas na Região Sul.

Da lista de alimentos industrializados disponíveis no supermercado selecionado, excluíramse aqueles para os quais não se aplica a legislação brasileira sobre rotulagem nutricional ${ }^{14} \mathrm{e} / \mathrm{ou}$ aqueles que não apresentavam gordura adicionada na composição, conforme detalhado na lista de ingredientes. Além disso, excluíram-se os produtos que não apresentavam a porção na informação nutricional; produtos em pó que não forneciam informações sobre rendimento, bem como aqueles que apresentavam a determinação do tamanho da porção somente por medida caseira.

Mediante formulário previamente testado, foram coletadas informações nos rótulos dos alimentos industrializados, incluindo a identificação do produto (tipo, nome comercial, sabor e marca), informações nutricionais (tamanho da porção em gramas ou mililitros e informação de conteúdo de gordura trans) e citação de componente com gordura trans na lista de ingredientes. Variações do mesmo produto alimentício em embalagens de tamanhos diferentes foram registradas como um novo produto, pois nem todos apresentavam porções similares.

A coleta de dados foi realizada em maio de 2010 por nutricionistas treinados, após obtenção de autorização da direção do supermercado. 


\section{Análise dos dados}

As informações foram duplamente digitadas em duas bases de dados distintas, posteriormente validadas para conferência de possíveis erros de digitação.

O tamanho da porção de cada produto alimentício foi categorizado de acordo com a adequação à RDC n ${ }^{\circ} 359 / 2003,{ }^{19}$ conforme demonstrado na tabela 1.

Tabela 1. Classificação do tamanho da porção em g ou ml declarada no rótulo em relação à porção recomendada pela RDC n³59/2003, legislação brasileira sobre rotulagem nutricional. Brasil, 2010.

\begin{tabular}{|c|c|c|}
\hline Classificação ${ }^{\mathrm{a}}$ & Significado & $\begin{array}{l}\text { Adequação segundo a } \\
\text { legislação brasileira }{ }^{\mathrm{b}}\end{array}$ \\
\hline$<70 \%$ & $\begin{array}{l}\text { Porção menor que } 70 \% \text { do tamanho da } \\
\text { porção recomendada em g ou ml }\end{array}$ & Inadequada \\
\hline $70-99 \%$ & $\begin{array}{c}\text { Porção até } 30 \% \text { menor que a porção } \\
\text { recomendada em g ou ml }\end{array}$ & Adequada \\
\hline $100 \%$ & $\begin{array}{l}\text { Porção idêntica à porção recomendada em } \\
\qquad \text { g ou ml }\end{array}$ & Adequada \\
\hline $101-130 \%$ & $\begin{array}{c}\text { Porção até } 30 \% \text { maior que a porção } \\
\text { recomendada em g ou ml }\end{array}$ & Adequada \\
\hline$>130 \%$ & $\begin{array}{l}\text { Porção maior que } 30 \% \text { do tamanho da } \\
\text { porção recomendada em g ou ml }\end{array}$ & Inadequada \\
\hline
\end{tabular}

${ }^{a}$ Classificação do tamanho da porção em $\mathrm{g}$ ou $\mathrm{ml}$ declarada no rótulo em relação à porção recomendada pela legislação. ${ }^{\mathrm{b}} \mathrm{RDC}$ n’$^{\circ} 359 / 2003$.

Para os alimentos classificados como prontos para o consumo, para os quais a legislação não estabelece o tamanho da porção de referência, foi calculada a densidade energética mediante a estimativa de quilocalorias para cada 100 gramas do produto correspondente. Esta variável foi posteriormente classificada em três categorias: $\leq 100$, 101-200 e > $200 \mathrm{kcal} / 100 \mathrm{~g}$. Para cada uma das categorias, foram calculados a mediana do tamanho da porção e o intervalo interquartil. 
Para determinar a presença de gordura trans nos alimentos industrializados, considerou-se a presença das seguintes denominações específicas de gorduras trans na lista de ingredientes: gordura vegetal hidrogenada, gordura vegetal parcialmente hidrogenada, óleo vegetal parcialmente hidrogenado e óleo vegetal hidrogenado. ${ }^{15,20}$ Ressalta-se que a gordura vegetal, quando completamente hidrogenada, é uma gordura sólida, que não contém ácidos graxos trans e não tem aplicação prática em alimentos. ${ }^{21}$ Em função disto, considerou-se que a denominação "gordura vegetal hidrogenada" indica a presença de gordura trans, ${ }^{21}$ passível de ser aplicada na produção de alimentos. Além disso, como gorduras, cremes vegetais e margarinas podem ou não conter gordura trans, estes itens foram incluídos como denominações alternativas da presença de gordura trans, com o objetivo de evitar a exclusão de alimentos passíveis de conter estes isômeros. ${ }^{22,23}$ Esses componentes foram definidos com base em evidências científicas que mostram, por análises físico-químicas, que estes ingredientes estão relacionados com maiores teores de gordura trans nos alimentos..$^{5,24,25}$

Foram identificados produtos que na informação nutricional reportaram não ter gordura trans, mas que apresentavam componente com gordura trans na lista de ingredientes, sendo assim estimada a prevalência de falsos negativos para a ausência de gordura trans.

Para análise das informações, os alimentos industrializados foram divididos em seis grandes grupos, conforme o tipo de alimento, baseados na classificação da RDC n⿳0 359/2003. ${ }^{19}$

- Grupo A: Produtos de panificação, cereais, leguminosas, raízes, tubérculos e derivados (biscoitos salgados, salgadinhos, batata palha, cereais matinais, pipocas de microondas, farofas, massas prontas secas, massas instantâneas, massas refrigeradas, massas de pizza, torradas, pães industrializados, batatas fritas congeladas, pães de queijo congelados, massas congeladas, polentas congeladas, pães de alho, sanduíches congelados, produtos de panificação).

- Grupo B: Derivados do leite (iogurtes, bebidas lácteas, queijos, requeijões, queijos cremosos).

- Grupo C: Derivados da carne (patês, empanados congelados, almôndegas congeladas, hambúrgueres).

- Grupo D: Óleos, gorduras e sementes oleaginosas (oleaginosas processadas, margarinas e gorduras vegetais, manteigas, chantillys).

- Grupo E: Açúcares e produtos que fornecem energia proveniente de carboidratos e gorduras (balas, gomas de mascar, doces de amendoim, chocolates, biscoitos doces, bolos prontos, sobremesas prontas, sorvetes, brigadeiros, granulados, pós para sorvetes, coberturas doces, cremes doces). 
- Grupo F: Molhos, temperos prontos, caldos, sopas e pratos preparados (temperos, pratos prontos a vácuo, lasanhas congeladas, pizzas congeladas, pratos prontos congelados, tortas congeladas).

Para análise da associação entre o tamanho da porção (ou a densidade energética para os produtos prontos para o consumo) e a presença de gordura trans, foram estimadas as prevalências, com os respectivos intervalos de confiança de 95\%. Foram utilizados os testes Qui-quadrado e Anova de tendência para variâncias heterogêneas para testar as associações, considerando o valor $\mathrm{p}<0,05$ como indicativo de significância estatística. A análise dos dados foi realizada no programa estatístico Stata versão 11.0 (StataCorp, College Station, TX, USA).

\section{Resultados}

Foram analisados 2.020 alimentos industrializados, sendo que 1.895 apresentavam porções definidas em gramas (pertencentes a todos os grupos); nos demais 125, as porções eram definidas em quilocalorias.

A análise da lista de ingredientes revelou que gordura vegetal hidrogenada (23\%) foi a principal denominação específica utilizada para notificar componente com gordura trans, enquanto gordura vegetal $(58,4 \%)$ e margarina $(13,4 \%)$ foram consideradas as principais denominações alternativas.

Com relação ao tamanho da porção, encontrou-se que 85,3\% dos alimentos industrializados (IC 95\% 83,6; 86,8) cumpriram com a normativa de variabilidade de $30 \%$ para mais e para menos do valor recomendado. Metade de todos os produtos analisados apresentava componente com gordura trans na lista de ingredientes, sendo que, em comparação ao reportado na informação nutricional, o percentual de falsos negativos foi de quase $40 \%$.

A presença de gordura trans na lista de ingredientes apresentou uma relação em "J" invertida, com o percentual de adequação ao tamanho da porção conforme a RDC n⿳⺈⿴囗十 $559 / 2003^{19}$ - ou seja, passou de apenas um terço entre aqueles produtos com o menor percentual de adequação para quase $75 \%$ entre aqueles no limite máximo da variabilidade permitida, enquanto pouco mais da metade dos produtos que excederam o limite permitido tinha gordura trans na lista de ingredientes. A associação entre a adequação do tamanho da porção e o percentual de falsos negativos apresentou padrão similar de incremento progressivo com posterior redução, dados destacados na tabela 2. 
Tabela 2. Associação entre o percentual de adequação à porção $(\mathrm{g})$ recomendada pela RDC no 359/2003 com a presença de gordura trans na lista de ingredientes dos alimentos industrializados e o percentual de falsos negativos ${ }^{\mathrm{a}}$. Florianópolis-SC, 2010.

\begin{tabular}{ccccccc}
\hline $\begin{array}{c}\text { Percentual de } \\
\text { adequação } \\
\text { à RDC } \\
\text { n'359/2003 }\end{array}$ & $\mathrm{N}$ & $\%$ & $\begin{array}{c}\text { \% Com } \\
\text { gordura } \\
\text { trans }\end{array}$ & IC 95\% & $\begin{array}{c}\text { \% Falsos } \\
\text { negativos }\end{array}$ & IC 95\% \\
\hline & & & & $\mathrm{P}<0,001^{*}$ & & $\mathrm{P}<0,001^{*}$ \\
$<70 \%$ & 203 & 10,7 & 36,0 & $(29,3 ; 42,9)$ & 30,5 & $(24,3 ; 37,4)$ \\
$70-99 \%$ & 152 & 8,0 & 46,1 & $(37,9 ; 54,3)$ & 38,2 & $(30,4 ; 46,4)$ \\
$100 \%$ & 1.392 & 73,5 & 52,6 & $(49,9 ; 55,2)$ & 39,0 & $(36,4 ; 41,6)$ \\
$101-130 \%$ & 73 & 3,9 & 74,0 & $(62,3 ; 83,5)$ & 50,7 & $(38,7 ; 62,6)$ \\
$>130 \%$ & 75 & 4,0 & 54,7 & $(42,7 ; 66,2)$ & 17,3 & $(9,6 ; 27,8)$ \\
\hline Total & 1.895 & 100,0 & 50,4 & $(48,1 ; 52,7)$ & 37,6 & $(35,4 ; 39,9)$ \\
\hline
\end{tabular}

* Teste de Qui-quadrado com correção de Yates.

IC 95\%: Intervalo com 95\% confiança.

aProdutos que na informação nutricional reportaram não ter gordura trans, mas que apresentavam este componente na lista de ingredientes.

A avaliação da presença de gordura trans de acordo com os grupos de alimentos industrializados (tabela 3) mostrou que mais de dois terços dos produtos classificados como açúcares e carboidratos (Grupo E) e mais da metade dos pães, biscoitos e massas (Grupo A) e dos pratos preparados (Grupo F) apresentavam gordura trans. Da mesma forma, se fez presente em cerca de um terço dos derivados da carne (Grupo C) e óleos e gorduras (Grupo D), enquanto apenas $5 \%$ dos derivados do leite (Grupo B) tinham este componente, sendo a diferença entre os grupos estatisticamente significativa $(\mathrm{p}<0,001)$. Nenhum padrão claro e nenhuma diferença estatisticamente significativa foi encontrado quando se testou a associação entre o percentual de adequação da porção e a presença de gordura trans na lista de ingredientes conforme os diferentes grupos de alimentos industrializados. 
Tabela 3. Associação entre o percentual de adequação à porção $(\mathrm{g})$ recomendada pela RDC no 359/2003 e o percentual de presença de gordura trans na lista de ingredientes, estratificados por grupos de alimentos industrializados. Florianópolis-SC, 2010.

\begin{tabular}{|c|c|c|c|c|c|}
\hline Grupo & $\begin{array}{c}\text { Percentual de } \\
\text { adequação à RDC } \\
\text { nº359/2003 }^{\circ}\end{array}$ & $\mathrm{N}$ & $\%$ & IC $95 \%$ & Valor-p \\
\hline \multirow{6}{*}{$\begin{array}{c}\text { Grupo A } \\
\text { Pães, biscoitos e } \\
\text { massas }\end{array}$} & $<70 \%$ & 50 & 56,0 & $(41,2 ; 70,0)$ & \multirow{6}{*}{$\mathrm{p}=0,101^{*}$} \\
\hline & $70-99 \%$ & 49 & 67,4 & $(52,4 ; 80,0)$ & \\
\hline & $100 \%$ & 463 & 56,2 & $(51,5 ; 60,7)$ & \\
\hline & $101-130 \%$ & 38 & 76,3 & $(59,7 ; 88,5)$ & \\
\hline & $>130 \%$ & 25 & 56,0 & $(34,9 ; 75,5)$ & \\
\hline & Total & 625 & 58,2 & $(54,3 ; 62,1)$ & \\
\hline \multirow{6}{*}{$\begin{array}{c}\text { Grupo B } \\
\text { Derivados do leite }\end{array}$} & $<70 \%$ & 93 & 6,5 & $(2,4 ; 13,5)$ & \multirow{6}{*}{$\begin{array}{c}\mathrm{p}= \\
0,204 * *\end{array}$} \\
\hline & $70-99 \%$ & 48 & 0,0 & $(--)$ & \\
\hline & $100 \%$ & 207 & 5,3 & $(2,7 ; 9,3)$ & \\
\hline & $101-130 \%$ & 1 & 0,0 & $(--)$ & \\
\hline & $>130 \%$ & 9 & 0,0 & $(--)$ & \\
\hline & Total & 358 & 4,8 & $(2,8 ; 7,5)$ & \\
\hline \multirow{6}{*}{$\begin{array}{c}\text { Grupo C } \\
\text { Derivados da } \\
\text { carne }\end{array}$} & $<70 \%$ & 12 & 58,3 & $(27,6 ; 84,8)$ & \multirow{6}{*}{$\mathrm{p}=0,147^{* *}$} \\
\hline & $70-99 \%$ & 13 & 38,5 & $(13,8 ; 68,4)$ & \\
\hline & $100 \%$ & 65 & 47,7 & $(35,1 ; 60,4)$ & \\
\hline & $101-130 \%$ & - & - & - & \\
\hline & $>130 \%$ & 7 & 0,0 & $(--)$ & \\
\hline & Total & 97 & 44,4 & $(34,2 ; 54,8)$ & \\
\hline \multirow{6}{*}{$\begin{array}{c}\text { Grupo D } \\
\text { Óleos e gorduras }\end{array}$} & $<70 \%$ & 1 & 100,0 & $(--)$ & \multirow{6}{*}{$\mathrm{p}=0,518^{* *}$} \\
\hline & $70-99 \%$ & - & - & - & \\
\hline & $100 \%$ & 69 & 37,7 & $(26,2 ; 50,1)$ & \\
\hline & $101-130 \%$ & - & - & - & \\
\hline & $>130 \%$ & 8 & 25,0 & $(0,31 ; 65,0)$ & \\
\hline & Total & 78 & 37,2 & $(26,5 ; 48,9)$ & \\
\hline
\end{tabular}




\begin{tabular}{|c|c|c|c|c|c|}
\hline Grupo & $\begin{array}{c}\text { Percentual de } \\
\text { adequação à RDC } \\
\text { n³59/2003 }\end{array}$ & $\mathrm{N}$ & $\%$ & IC $95 \%$ & Valor-p \\
\hline \multirow{6}{*}{$\begin{array}{c}\text { Grupo E } \\
\text { Açúcares e } \\
\text { carboidratos }\end{array}$} & $<70 \%$ & 45 & 68,9 & $(53,3 ; 81,8)$ & \multirow{6}{*}{$\mathrm{p}=0,103^{*}$} \\
\hline & $70-99 \%$ & 37 & 73,0 & $(55,8 ; 86,2)$ & \\
\hline & $100 \%$ & 546 & 69,6 & $(65,5 ; 73,4)$ & \\
\hline & $101-130 \%$ & 34 & 73,5 & $(55,6 ; 87,1)$ & \\
\hline & $>130 \%$ & 23 & 43,5 & $(23,1 ; 65,5)$ & \\
\hline & Total & 685 & 69,1 & $(65,4 ; 72,5)$ & \\
\hline \multirow{6}{*}{$\begin{array}{c}\text { Grupo F } \\
\text { Pratos prontos } \\
\text { para o consumo }\end{array}$} & $<70 \%$ & 2 & 0,0 & $(--)$ & \multirow{6}{*}{$\mathrm{p}=0,084^{* *}$} \\
\hline & $70-99 \%$ & 5 & 100,0 & $(--)$ & \\
\hline & $100 \%$ & 42 & 57,1 & $(40,9 ; 72,2)$ & \\
\hline & $101-130 \%$ & - & - & - & \\
\hline & $>130 \%$ & 3 & 0,0 & $(--)$ & \\
\hline & Total & 52 & 55,8 & $(41,3 ; 69,5)$ & \\
\hline
\end{tabular}

* Teste de Qui-quadrado com correção de Yates.

** Teste de Qui-quadrado exato de Fisher.

IC $95 \%$ : Intervalo com $95 \%$ confiança.

Na tabela 4, pode-se observar que o percentual de falsos negativos ultrapassou $50 \%$ no grupo de pratos preparados e dos açúcares e carboidratos, seguido por pães, biscoitos e massas. Por sua vez, o percentual de falsos negativos foi de um terço nos produtos classificados como derivados da carne e os óleos e gorduras, sendo de apenas $4 \%$ nos derivados do leite $(\mathrm{p}<0,001)$. No grupo dos pães, biscoitos e massas, mais da metade dos produtos com tamanho de porção adequada foram identificados como falsos negativos $(\mathrm{p}<0,001)$. No grupo dos derivados da carne, o maior percentual de falsos negativos aconteceu entre os produtos com o tamanho da porção menor que $70 \%$ do valor recomendado $(\mathrm{p}=0,035)$. Os demais grupos não apresentaram diferença estatisticamente significativa entre o percentual de falsos negativos e o percentual de adequação à porção da resolução. 
Tabela 4. Associação entre o percentual de adequação à porção (g) recomendada pela RDC no 359/2003 e o percentual de falsos negativos ${ }^{a}$ em relação à presença de gordura trans estratificados por categorias de alimentos industrializados. Florianópolis-SC, 2010.

\begin{tabular}{|c|c|c|c|c|c|}
\hline Grupo & $\begin{array}{c}\text { Percentual de } \\
\text { adequação à RDC } \\
\text { n³59/2003 }\end{array}$ & $\mathrm{N}$ & $\%$ & IC $95 \%$ & Valor-p \\
\hline \multirow{6}{*}{$\begin{array}{c}\text { Grupo A } \\
\text { Pães, biscoitos e } \\
\text { massas }\end{array}$} & $<70 \%$ & 50 & 46,0 & $(31,8 ; 60,6)$ & \multirow{6}{*}{$\mathrm{p}=0,001^{*}$} \\
\hline & $70-99 \%$ & 49 & 59,2 & $(44,4 ; 73,0)$ & \\
\hline & $100 \%$ & 463 & 40,2 & $(35,6 ; 44,7)$ & \\
\hline & $101-130 \%$ & 38 & 55,2 & $(38,2 ; 71,3)$ & \\
\hline & $>130 \%$ & 25 & 12,0 & $(2,5 ; 31,2)$ & \\
\hline & Total & 625 & 41,9 & $(38,0 ; 45,9)$ & \\
\hline \multirow{6}{*}{$\begin{array}{c}\text { Grupo B } \\
\text { Derivados do leite }\end{array}$} & $<70 \%$ & 93 & 6,5 & $(2,4 ; 13,5)$ & \multirow{6}{*}{$\mathrm{p}=0,139 * *$} \\
\hline & $70-99 \%$ & 48 & 0,0 & $(--)$ & \\
\hline & $100 \%$ & 207 & 4,4 & $(2,0 ; 8,0)$ & \\
\hline & $101-130 \%$ & 1 & 0,0 & $(--)$ & \\
\hline & $>130 \%$ & 9 & 0,0 & $(--)$ & \\
\hline & Total & 358 & 4,2 & $(2,4 ; 6,8)$ & \\
\hline \multirow{6}{*}{$\begin{array}{c}\text { Grupo C } \\
\text { Derivados da } \\
\text { carne }\end{array}$} & $<70 \%$ & 12 & 58,3 & $(27,6 ; 84,8)$ & \multirow{6}{*}{$\mathrm{p}=0,035^{* *}$} \\
\hline & $70-99 \%$ & 13 & 7,7 & $(0,1 ; 36,0)$ & \\
\hline & $100 \%$ & 65 & 36,9 & $(25,2 ; 49,8)$ & \\
\hline & $101-130 \%$ & - & - & - & \\
\hline & $>130 \%$ & 7 & 0,0 & $(--)$ & \\
\hline & Total & 97 & 33,0 & $(23,8 ; 43,3)$ & \\
\hline \multirow{6}{*}{$\begin{array}{c}\text { Grupo D } \\
\text { Óleos e gorduras }\end{array}$} & $<70 \%$ & 1 & 100,0 & $(--)$ & \multirow{6}{*}{$\mathrm{p}=0,300 * *$} \\
\hline & $70-99 \%$ & - & - & - & \\
\hline & $100 \%$ & 69 & 33,3 & $(22,3 ; 45,7)$ & \\
\hline & $101-130 \%$ & - & - & - & \\
\hline & $>130 \%$ & 8 & 12,5 & $(0,3 ; 52,6)$ & \\
\hline & Total & 78 & 32,1 & $(21,9 ; 43,6)$ & \\
\hline
\end{tabular}




\begin{tabular}{|c|c|c|c|c|c|}
\hline Grupo & $\begin{array}{c}\text { Percentual de } \\
\text { adequação à RDC } \\
\text { n³59/2003 }\end{array}$ & $\mathrm{N}$ & $\%$ & IC $95 \%$ & Valor-p \\
\hline \multirow{6}{*}{$\begin{array}{c}\text { Grupo E } \\
\text { Açúcares e } \\
\text { carboidratos }\end{array}$} & $<70 \%$ & 45 & 55,6 & $(39,9 ; 70,3)$ & \multirow{6}{*}{$\mathrm{p}=0,442 *$} \\
\hline & $70-99 \%$ & 37 & 62,2 & $(44,7 ; 77,5)$ & \\
\hline & $100 \%$ & 546 & 50,7 & $(46,4 ; 55,0)$ & \\
\hline & $101-130 \%$ & 34 & 47,1 & $(29,7 ; 64,8)$ & \\
\hline & $>130 \%$ & 23 & 39,1 & $(19,7 ; 61,4)$ & \\
\hline & Total & 685 & 51,1 & $(47,3 ; 54,9)$ & \\
\hline \multirow{6}{*}{$\begin{array}{c}\text { Grupo } \mathrm{F} \\
\text { Pratos prontos } \\
\text { para o consumo }\end{array}$} & $<70 \%$ & 2 & 0,0 & $(--)$ & \multirow{6}{*}{$\mathrm{p}=0,083^{* *}$} \\
\hline & $70-99 \%$ & 5 & 100,0 & $(--)$ & \\
\hline & $100 \%$ & 42 & 57,1 & $(40,9 ; 72,2)$ & \\
\hline & $101-130 \%$ & - & - & - & \\
\hline & $>130 \%$ & 3 & 0,0 & $(--)$ & \\
\hline & Total & 52 & 55,8 & $(41,3 ; 69,5)$ & \\
\hline
\end{tabular}

* Teste de Qui-quadrado com correção de Yates.

** Teste de Qui-quadrado exato de Fisher.

IC 95\%: Intervalo com 95\% Confiança.

a Produtos que na informação nutricional reportaram não ter gordura trans, mas que apresentavam componente com gordura trans na lista de ingredientes.

Para os produtos classificados como prontos para o consumo (tabela 5), a densidade energética esteve inversamente relacionada com o tamanho da porção. A presença de gordura trans foi maior nos produtos com densidade energética entre $101-200 \mathrm{kcal} / 100 \mathrm{~g}$, diminuindo nos produtos com densidade $\leq 100 \mathrm{kcal} / 100 \mathrm{~g}$, sendo menor, ainda, naqueles $>200 \mathrm{kcal} / 100 \mathrm{~g}(\mathrm{p}<0,001)$. Por sua vez, houve uma relação inversa entre a densidade energética e o percentual de falsos negativos, com tendência estatisticamente significativa $(\mathrm{p}=0,049)$, bem como houve uma relação inversa entre a densidade energética e a mediana do tamanho da porção em gramas $(p<0,001)$. 
Tabela 5. Associação entre a densidade energética (kcal/100g) e o tamanho da porção, presença de gordura trans na lista de ingredientes e os percentuais de falsos negativos. ${ }^{a}$ Florianópolis-SC, 2010 ( $\mathrm{N}=125)$.

\begin{tabular}{|c|c|c|c|c|c|c|}
\hline \multirow{3}{*}{$\begin{array}{l}\text { Quilocalorias } \\
\text { por } 100 \mathrm{~g}\end{array}$} & \multirow{3}{*}{$\mathrm{N}$} & \multirow{3}{*}{$\begin{array}{l}\text { Tamanho da porção }(\mathrm{g}) \\
\text { Mediana } \\
\text { (intervalo interquartil) }\end{array}$} & \multirow{2}{*}{\multicolumn{2}{|c|}{$\begin{array}{c}\text { Presença de gordura } \\
\text { trans }\end{array}$}} & \multirow{2}{*}{\multicolumn{2}{|c|}{ Falsos negativos }} \\
\hline & & & & & & \\
\hline & & & $\%$ & IC $95 \%$ & $\%$ & IC $95 \%$ \\
\hline & & $\mathrm{P}<0,001^{*}$ & & $\mathrm{P}<0,001^{* *}$ & & $\mathrm{P}=0,049 \dagger$ \\
\hline$\leq 100 \mathrm{kcal}$ & 14 & $328(250 ; 420)$ & 28,6 & $(8,3 ; 58,1)$ & 28,6 & $(8,3 ; 58,1)$ \\
\hline $101-200 \mathrm{kcal}$ & 54 & $313(100 ; 325)$ & 57,4 & $(43,2 ; 70,7)$ & 16,7 & $(7,9 ; 29,2)$ \\
\hline$>200 \mathrm{kcal}$ & 57 & $100(58 ; 125)$ & 21,1 & $(11,3 ; 33,8)$ & 8,8 & $(76,3 ; 94,9)$ \\
\hline \multicolumn{7}{|c|}{ * Teste de ANOVA de tendência para variâncias heterogêneas } \\
\hline \multicolumn{7}{|c|}{ ** Teste de Qui-quadrado de heterogeneidade com correção de Yates. } \\
\hline \multicolumn{7}{|c|}{$†$ Teste de Qui-quadrado de tendência. } \\
\hline \multicolumn{7}{|c|}{ IC 95\%: Intervalo com 95\% confiança. } \\
\hline
\end{tabular}

\section{Discussão}

No estudo observou-se que mais da metade dos alimentos industrializados analisados apresentavam algum componente com gordura trans, segundo a lista de ingredientes. Assim, este resultado revela que possivelmente as indústrias não estão cumprindo o acordo firmado com a OMS para redução da gordura trans nos produtos industrializados. ${ }^{11}$ Estudo realizado por Monge-Rojas et al. ${ }^{26}$ com o objetivo de investigar o cumprimento desse acordo em 12 indústrias, recebeu respostas de apenas três (25\%). Segundo os autores, esse acordo parece não garantir e não oferecer informações suficientes para o monitoramento da redução da gordura trans industrial na América Latina e Caribe.

Encontrou-se uma variedade de denominações para declaração de componente com gordura trans na lista de ingredientes. Destas, mais da metade foram denominadas de "alternativas", pois não havia certeza quanto ao tipo de gordura utilizada. Esse fato pode confundir o consumidor no momento de identificar se o produto contém ou não gordura trans.

Verificou-se que foram os grupos "pães, biscoitos e massas" e "açúcares e carboidratos" que apresentaram maior percentual de gordura trans na lista de ingredientes. Tais achados são preocupantes, visto que é elevado o consumo desses produtos pela população brasileira., ${ }^{2,22}$ Monteiro et al. ${ }^{22}$ demonstram a mudança no padrão alimentar brasileiro ao compararem os dados das Pesquisas de Orçamento Familiar de 2002-03 e 2008-09, ${ }^{2}$ notando aumento de compra de $40 \%$ 
para refeições prontas e misturas industrializadas, bem como de 10\% para biscoitos. Destaca-se, ainda, que alguns estudos mostram que os alimentos com maior quantidade de gordura trans são, geralmente, mais baratos e mais acessíveis à população de baixa renda., ${ }^{4,827}$

No que se refere aos grupos $\mathrm{B}$ e C, alimentos derivados do leite e derivados da carne, respectivamente, observou-se a presença de gordura trans na lista de ingredientes em $5 \%$ dos produtos do primeiro grupo e em um terço dos produtos do segundo grupo. Ressalta-se que nos alimentos pertencentes a esses grupos a gordura trans pode estar presente pela formação natural desta gordura, por meio do processo de bio-hidrogenação realizado pela microbiota do rúmen dos animais. ${ }^{21,28}$ Entretanto, caso fosse somente a notificação da gordura trans naturalmente formada, não haveria a presença de gordura trans na lista de ingredientes, pois ela somente apareceria na informação nutricional. Assim, os achados do presente estudo revelam a adição de gordura trans industrial em alimentos que já possuem essa gordura naturalmente. É importante considerar que, durante o processo de produção natural de gordura trans, há a formação do ácido linoleico conjugado (CLA), que vem sendo associado a benefícios para a saúde, embora os estudos ainda não sejam conclusivos .,29-31

Constatou-se que os alimentos industrializados analisados que cumpriam o preconizado pela legislação vigente em relação ao tamanho da porção declarada no rótulo representaram o maior percentual, considerando a margem de $30 \%$ para mais e para menos. ${ }^{15}$ Contudo, destaca-se que $15 \%$ $(n=278)$ deles estavam inadequados. Estes dados corroboram Lobanco et al., ${ }^{32}$ que identificaram inconformidades na informação nutricional da maioria dos alimentos industrializados analisados, demonstrando violação das disposições das resoluções sobre rotulagem e direitos garantidos pelo Código de Defesa do Consumidor. ${ }^{33}$

Destaca-se, contudo, que foram elevados tanto o percentual de presença de gordura trans segundo a lista de ingredientes, quanto o percentual de falsos negativos, mesmo nos alimentos industrializados em que o tamanho da porção estava de acordo com a legislação.

$\mathrm{Na}$ análise por grupo, embora o percentual de falsos negativos fosse mais elevado (40-55\%) em três dos seis grupos avaliados (açúcares e carboidratos, pratos preparados e pães, biscoitos e massas), manteve-se também maior percentual de falsos negativos entre os produtos com tamanho de porção adequada. Portanto, os resultados sugerem que o tamanho da porção recomendada pela Resolução 359/2003 ${ }^{19} \mathrm{em}$ muitos casos, é insuficiente para revelar a presença de gordura trans na informação nutricional, podendo comprometer a interpretação do consumidor sobre a presença desse tipo de gordura no alimento.

Resultado semelhante foi encontrado em estudo realizado nos Estados Unidos, em que pães, salgadinhos e barras de cereais apresentaram tanto maior percentual de alimentos com a declaração de "0 grama de gordura trans" quanto o maior percentual de alimentos com conteúdo de gordura trans conforme a informação nutricional. ${ }^{34}$ Considerando que nesse país os alimentos industrializados com conteúdo de gordura trans menor que $0,5 \mathrm{~g}$ por porção podem ser declarados 
como "zero trans", esses pesquisadores sugeriram que a indústria alimentícia pode estar usufruindo da fragilidade da legislação norte-americana para realizar propaganda de seus produtos como "zero trans", mesmo quando tais alimentos contenham gordura trans. ${ }^{23}$ Essa mesma hipótese pode ser levantada no presente estudo com relação à legislação brasileira.

Na análise da rotulagem nutricional dos pratos preparados, observou-se que os alimentos com menor densidade energética apresentavam percentual intermediário de presença de gordura trans na lista de ingredientes, porém o maior percentual de falsos negativos. Considerando que é provável que os consumidores percebam como mais saudáveis aqueles alimentos que apresentam menores valores calóricos na rotulagem, ${ }^{35}$ encontrou-se que tais pratos preparados - embora aparentando ser mais saudáveis por terem baixa densidade energética - apresentam menor fidedignidade na informação sobre gordura trans. Por sua vez, o grupo dos pratos preparados com maior densidade energética, mesmo tendo menor percentual de produtos com gordura trans e de falsos negativos, foram os produtos que apresentaram menor tamanho da porção. Isto demonstra que a não determinação legal de tamanho de porção em gramas para este grupo de alimentos permite que os fabricantes rotulem um tamanho de porção que possa favorecer o produto para a notificação de ingredientes considerados não saudáveis. Nesse sentido, Ferreira \& Lanfer-Marquez ${ }^{36}$ ressaltam a importância da definição de uma porção recomendada para esse grupo, por se tratar de alimentos industrializados cada vez mais presentes na alimentação da população.

Nesta pesquisa, demonstrou-se a implicação do uso da informação nutricional por porção para identificar a fidedignidade da informação nutricional da gordura trans oferecida aos consumidores. Segundo a literatura, o tamanho da porção pode vir a comprometer a notificação de outros nutrientes com efeitos adversos sobre a saúde dos indivíduos, tais como sódio e gordura saturada ${ }^{30}$. Adicionalmente, a variabilidade permitida pela legislação brasileira pode afetar o objetivo de promover uma alimentação saudável aos consumidores. De acordo com Garsetti et al., ${ }^{37}$ apesar de a informação nutricional por porção ser importante em função de os alimentos serem consumidos em variadas porções, se fosse acrescentada a informação nutricional por 100 gramas, os consumidores poderiam ser mais bem informados sobre a quantidade dos nutrientes presentes nos alimentos, facilitando a comparação entre produtos de diferentes marcas.

Dentre as limitações do presente estudo, cita-se o uso apenas das informações presentes nos rótulos, não sendo feitas análises físico-químicas, nem a pesagem dos alimentos. Entretanto, analisaram-se as informações disponíveis ao consumidor nos rótulos, as únicas de que ele dispõe no momento da compra para orientar as escolhas alimentares. Portanto, considerando o direito do consumidor e o objetivo da rotulagem como uma política pública de saúde, essas informações devem ter sua fidedignidade garantida pela empresa fabricante e serem passíveis de avaliação à luz da legislação vigente. Outra possível limitação deste estudo foi a inclusão de alimentos industrializados de um único supermercado. Entretanto, o local pesquisado faz parte 
de uma grande rede de supermercados e muitos dos alimentos industrializados avaliados são comercializados em todo o país.

\section{Conclusão}

Com o presente estudo, foi possível identificar um elevado percentual de alimentos industrializados cuja informação nutricional declarava não ter gordura trans, mas que apresentavam gordura fonte na lista de ingredientes. Essa observação foi verdadeira mesmo para produtos que seguem a porção recomendada pela legislação. Os grupos dos pães, biscoitos e massas e o grupo dos açúcares e carboidratos, bem como o dos pratos preparados foram os destacados neste quesito.

Assim, o tamanho de porção revelou ser insuficiente para notificar a presença de gordura trans nos alimentos, sendo necessário consultar a lista de ingredientes.

Diante do exposto, reforça-se que esses achados sejam considerados para a revisão da legislação brasileira sobre a notificação da gordura trans na rotulagem de alimentos, bem como que sejam promovidas ações educativas para informar apropriadamente os consumidores quanto à presença de gordura trans notificada no rótulo dos alimentos industrializados.

\section{Agradecimentos}

A Coordenação de Aperfeiçoamento de Pessoal de Nível Superior (CAPES) pela concessão de: Bolsa de Mestrado para Nathalie Kliemann. Conselho Nacional de Desenvolvimento Científico e Tecnológico (CNPq) - Bolsa de Desenvolvimento Tecnológico Industrial (DTI 3) para Bruna Maria Silveira e Bolsa de Iniciação Científica (PIBIC) para Mariana Vieira dos Santos Kraemer.

\section{Referências}

1. Popkin BM. Global nutrition dynamics: the world is shifting rapidly toward a diet linked with noncommunicable diseases. Am. J. Clin. Nutr. 2006; 84(2):289-298.

2. Instituto Brasileiro de Geografia e Estatística. Pesquisa de orçamentos familiares 2008-2009: aquisição alimentar domiciliar per capita. Rio de Janeiro: IBGE; 2010. 272 p.

3. Monteiro CA, Cannon G, Levy RB, Claro R, Moubarac JC, Martins AP, et al. The food system. Ultra-processing. The big issue for nutrition, disease, health, well-being. J. World Public Health Nutr. Assoc. [Internet] 2012; 3(12).

4. Castro MA, Barros RR, Bueno MB, César CLG, Fisberg RM. Trans fatty acids intake among the population of the city of São Paulo, Southeastern Brazil. Rev. Saúde Pública 2009; 43(6):991-997. 
5. Chiara VL, Sichieri R, Carvalho TSF. Teores de ácidos graxos trans de alguns alimentos consumidos no Rio de Janeiro. Rev. Nutr. 2003; 16(2):227-233.

6. Chavarro JE, Rich-Edwards JW, Rosner BA, Willett WC. Dietary fatty acid intakes and the risk of ovulatory infertility. Am. J. Clin. Nutr. 2007; 85(1):231-237.

7. Costa AGV, Bressan J, Sabarense CM. Ácidos graxos trans: alimentos e efeitos na saúde. Arch. Latinoam. Nutr. 2006; 56(1):12-21.

8. Remig V, Franklin BSM, Kostas G, Nece T, Street JC. Trans fats in America: a review of their use, consumption, health implications, and regulation. J. Am. Diet Assoc. 2010; 110(4):585-592.

9. World Health Organization. United Nations. Global strategy on diet, physical activity and health: list of all documents and publications. Fifty-seventh World Health Assembly, A57/9. Geneva: WHO; 2004.

10. World Health Organization. Follow-up to the political declaration of the high-level meeting of the General Assembly on the Prevention and Control of Non-communicable Diseases. Sixty-sixth World Health Assembly. Geneva: WHO; 2013.

11. Organização Pan-Americana da Saúde. Américas livres de gorduras trans. Rio de Janeiro: OPAS; 2008. Acesso em: 31 jan. 2013. Disponível em: http://189.28.128.100/dab/docs/portaldab/documentos/ nota_imprensa_gorduras_trans.pdf

12. Malik VS, Willett WC, Hu FB. Global obesity: trends, risk factors and policy implication. Nat. Rev. Endocrinol. 2013; 9(1):13-27.

13. Brownell KD, Pomeranz JL. The trans-fat ban: food regulation and long-term health. N. Engl. J. Med. 2014; 370(19):1773-1775.

14. Brasil. Resolução RDC no 360, de 23 de dezembro de 2003. A Diretoria Colegiada da ANVISA/MS aprova o regulamento técnico sobre rotulagem nutricional de alimentos embalados. Diário Oficial da União 26 dez. 2003.

15. Proença RPC, Silveira BM. Recomendações de ingestão e rotulagem nutricional de gordura trans em alimentos industrializados brasileiros: análise de documentos oficiais. Rev. Saúde Pública 2012; 46(5):923-928.

16. Hissanaga VM, Pastore JA, Proença RPC. Gordura trans: experiências de controle em unidades produtoras de refeições. Nutrição em Pauta 2010; 104(18):4-9.

17. Hissanaga VM, Block JM, Proença RPC. Development of a Method for Controlling Trans Fatty Acids in Meals - MCTM. J. Culinary Sci. Technology 2012; 10:1-18.

18. Gagliardi ACM, Mancini Filho J, Santos RD. Perfil nutricional de alimentos com alegação de zero gordura trans. Rev. Assoc. Med. Bras. 2009; 55(1):50-53.

19. Brasil. Resolução RDC no 359, de 23 de dezembro de 2003. A Diretoria Colegiada da ANVISA/MS aprova regulamento técnico de porções de alimentos embalados para fins de rotulagem nutricional. Diário Oficial União 26 dez. 2003.

20. Silveira BM, Gonzalez-Chica DA, Costa Proença RP. Reporting of trans-fat on labels of Brazilian food products. Public Health Nutr. 2013; 16(12):1-8.

21. Martin CA, Matshushita M, Souza NE. Ácidos graxos trans: implicações nutricionais e fontes na dieta. Rev. Nutr. 2004; 17(3):361-368. 
22. Monteiro CA, Levy RB, Claro RM, Castro IR, Cannon G. Increasing consumption of ultra-processed foods and likely impact on human health: evidence from Brazil. Public Health Nutr. 2011; 14(1):5-13.

23. Scheeder MRL. About the trans-(hi) story: how did trans fatty acids enter the human food chain. J. Am. Oil Chem. Soc. 2007; 18(2):133-135.

24. Aued-Pimentel S, Caruso MF, Cruz JMM, Kumagai EE, Corrêa DUA. Ácidos graxos saturados versus ácidos graxos trans em biscoitos. R. Inst. Adolfo Lutz 2003; 62(2):131-137.

25. Wagner KH, Plasser E, Proell C, Kanzler S. Comprehensive studies on the trans fatty acid content od Austrian foods: convenience products, fast food and fats. Food Chemistry 2008; 108(3):1054-1060.

26. Monge-Rojas R, Colón-Ramos U, Jacoby E, Mozaffarian D. Voluntary reduction of trans-fatty acids in Latin America and the Caribbean: current situation. Rev. Panam. Salud Publica 2011; 29(2):126-129.

27. Silveira BM, Kliemann N, Silva DP, Colussi CF, Proença RP. Availability and price of food products with and without trans fatty acids in food stores around elementary schools in low- and mediumincome neighborhoods. Ecol. Food Nutr. 2013; 52(1):63-75.

28. Mozaffarian D, Katan MB, Ascherio A, Stampfer MJ, Willett WC. Trans fatty acids and cardiovascular disease. N. Engl. J. Med. 2006; 354(15):1601-1613

29. Agueda M, Ángeles M, Martínez JF. Efecto del ácido linoleico conjugado (CLA) sobre el perfil lipídico en humanos. Arch. Latinoam. Nutr. 2009; 59(3):245-252.

30. Santos-Zago LF, Botelho AP, Oliveira AC. Os efeitos do ácido linoléico conjugado no metabolismo animal: avanço das pesquisas e perspectivas para o futuro. Rev. Nutr. 2008; 21(2):195-221.

31. Tanaka, K. Occurrence of conjugated linoleic acid in ruminant products and its physiological function. Anim. Sci. J. 2005; 76(4):291-303.

32. Lobanco CM, Vedovato GM, Cano CB, Bastos DHM. Fidedignidade de rótulos de alimentos comercializados no município de São Paulo, SP. Rev. Saúde Pública 2009; 43(3):499-505.

33. Brasil. Lei no 8.078, de 11 de setembro de 1990: dispõe sobre a proteção do consumidor e dá outras providências. Diário Oficial da União 11. set. 1990.

34. Brandt M, Moss J, Ferguson M. The 2006-2007 Food Label and Package Survey (FLAPS): nutrition labeling, trans fat labeling. J. Food Compost Anal. 2009; 22(1):S74-S77.

35. Steenhuis IHM, Vermeer, WM. Portion size: review and framework for interventions. Int. J. Behav. Nut. Phys. Act. 2009; 6(58):1-10.

36. Ferreira AB, Lanfer-Marquez UM. Legislação brasileira referente à rotulagem nutricional de alimentos. Rev. Nutr. 2007; 20(1):83-93.

37. Garsetti M, De Vries J, Smith M, Amosse A, Rolf-Pedersen N. Nutrient profiling schemes: overview and comparative analysis. Eur. J. Nutr. 2007; 46(2):15-28.

Recebido: $23 / 9 / 2014$

Revisado: $16 / 12 / 2014$

Aprovado: $14 / 1 / 2015$ 